\title{
Research on a kind of human motion state monitoring device
}

\author{
Wang Jin ${ }^{1, a^{*}}$, Liu Yanfei ${ }^{2, b}$ \\ ${ }^{1}$ Chongqing Information Technology College, Chongqing 404000, China \\ ${ }^{2}$ Chongqing Public Security Bureau, Chongqing 400050, China \\ a77282901@qq.com, ${ }^{b}$ filket@163.com
}

Keywords: Movement ; monitoring ; sensor ;

Abstract. As people's living standards improve, people have higher requirements on the quality of life and health of human body. Exercise is an important way to keep healthy. The amount of exercise of different degree will have different effects on the health of human body. Real-time monitoring and recording the movement of the human body, which Can provide scientific basis for the study of the relationship between human movement and health. This paper introduces a design with display and warning function, and low power dissipation function of the human body movement monitoring instrument. Real-time monitoring of human movement condition is very meaningful.

\section{Introduction}

The economic and social development significantly improved people's living standards, the improvement of life level and let people more and more attention to health, reasonable exercise is one of the important ways to keep people healthy. Real-time monitoring and record on the motion state of human body, can provide scientific basis for the study of the relationship between human movement and health. So the real-time monitoring for human motion status is very meaningful.

In recent years, the domestic scholar research work of human condition monitoring in various fields, such as in the field of aviation development pilot physiological status monitoring system for monitoring the physiological state of the driver[1]. In the medical field by measuring the body surface micro climate, heart rate monitor the physiological conditions of patients [2-3].Sports health care field, through the detection of gas metabolism analysis of human energy consumption status [4-5]. These works on monitoring index of human motion are relatively single, and most of the high cost, the operation process is more complex, the lack of flexibility, so easy to restricted application. This paper describes the design of a monitoring system for human motion, which have rich measurement function, friendly interface, low power performance. The system integrates three-dimensional acceleration sensor and the temperature and humidity sensor as information acquisition device, three-dimensional acceleration sensor is used to measure the human motion steps, through the relevant physiological parameters to calculate the number of steps and human body movement speed, distance, average energy consumption etc. Temperature and humidity sensor can be used for real-time temperature and humidity measurement in the process of human motion. This human motion monitoring system can display in the process of movement parameters of human body in real time, and early warning to the human body excessive exercise behavior, suggesting that movement to avoid excessive movement. As well as the system has low power consumption.

\section{The device structure and function design}

Human motion monitoring device is designed in this paper includes seven parts: micro controller, keyboard module, sensing a parameter acquisition module, storage module, liquid crystal display module and power supply module. As shown in Fig1. 


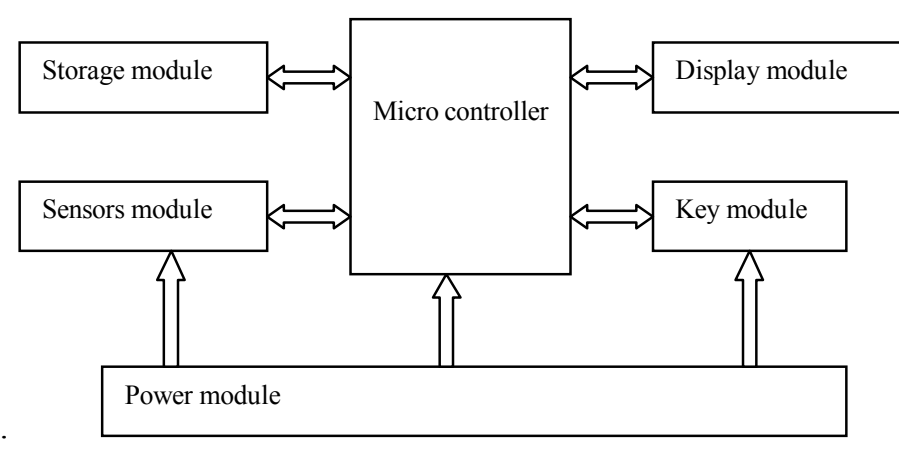

Fig.1 Device structure chart of human

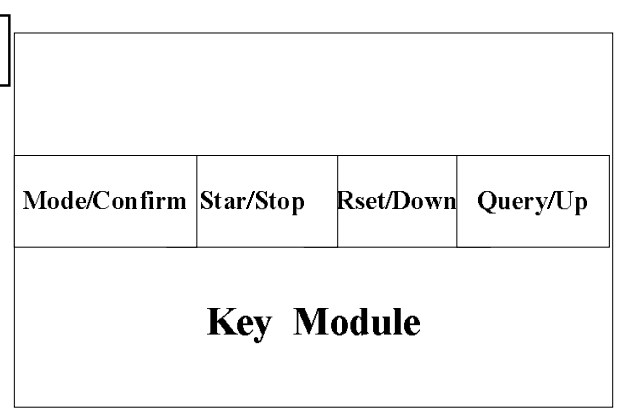

Fig.2 Key Module

The key module includes four functional buttons, which are mode/confirm button, star/stop button, reset/down button and query/add button, shown in Fig 2. These keys are mainly used to implement the parameter initialization, information display, monitoring control and so on.

The module includes two kinds of digital sensors to acquire sensing parameters, The one is a three-dimensional acceleration sensor, which is used to measure the human gait; the other is a digital temperature and humidity sensor, which is used to measure the human body temperature and humidity in the course of movement.

The sensor module to obtain human motion state information, through the control processing unit processing and output the statement, through the key output of various human motion state information. Display module mainly includes information about the time of begins to movement, real-time temperature and humidity of human body in motion, real-time human body water loss, the human body movement total steps, human body average speed and so on. In the process of human motion if the amount of exercise beyond the normal range, the device will remind the user to rest. When a new round of monitoring user start, the device will save the historical date, which can provide users with a contrast. The power management module is responsible for each module can provide power supply.

\section{Hardware design}

A control and processor design

According to the human body movement monitoring system structure and function module design, the hardware core control module selects the SHC6601 SOC. SHCC6601 is an ultra-low power MCU, which integrated enhanced 80C251 control core ,4 general purpose 16 bit timer / counter, buzzer frequency generator, $32 \mathrm{~K}$ bytes of flash memory, 58 general-purpose $\mathrm{I} / \mathrm{O}$ pins, and communication interface contains 2 UART, $1 \mathrm{SPI}$, and $1 \mathrm{I} 2$, so the interface resources are very rich, Requires only a small amount of peripheral components that can drive system, Fig. 3 is a typical application circuit of SHC6601 design[6].P54 as the buzzer design of human motion monitoring device.
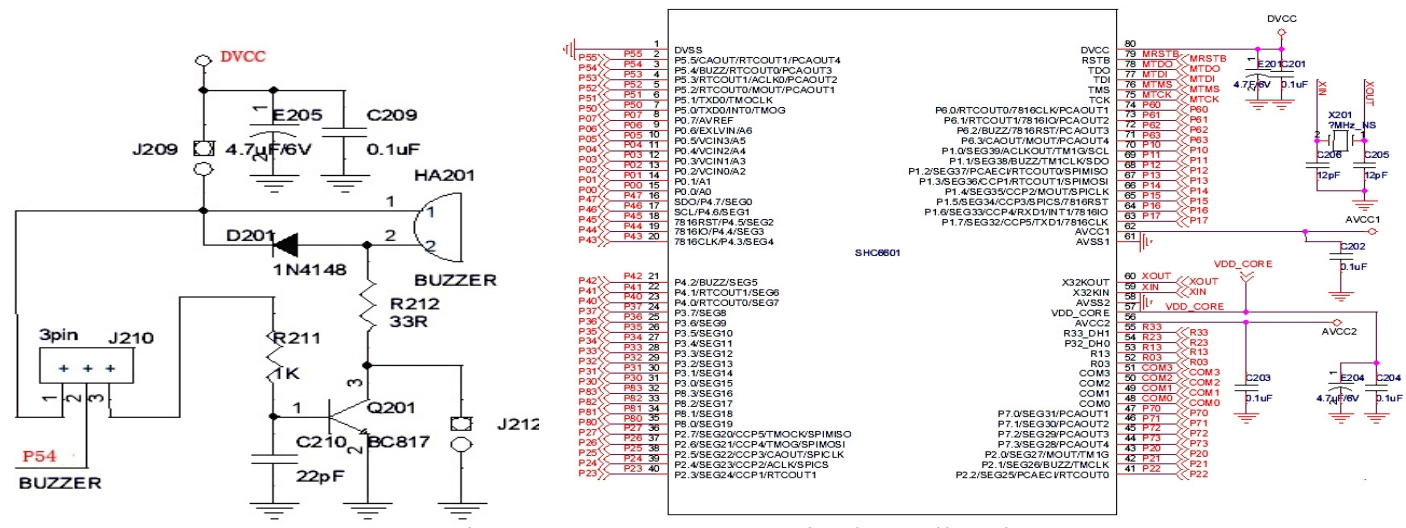

Fig..3 SHCC6601 typical application 


\section{B Interface circuit design}

Interface circuit design mainly includes the interface design between SHCC6601 and sensors. This paper selects CMA3000 to measure the human step, CMA3000 is a three-dimensional acceleration sensor digital sensor of Finland VTI company, through the SPI interface for communication between CMA3000 and SHCC6601, as shown in Fig. 4 below: The 4 SPI ports and interrupt port of CMA3000 are respectively connected with P12-P16 interface of SHCC6601. Between DVCC and GND connected to a decoupling capacitor.

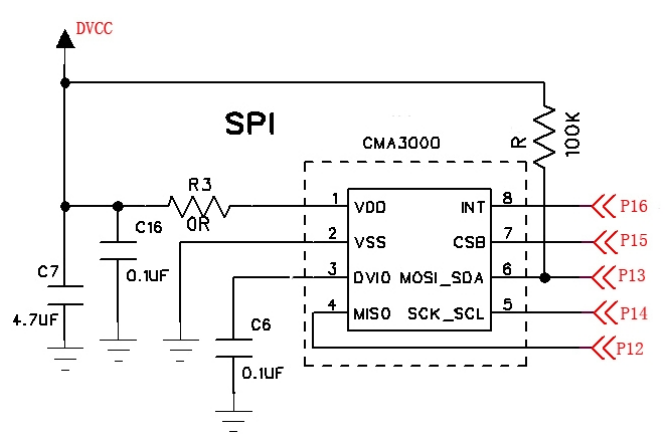

Fig.4 The interface design between SHCC6601and CMA3000

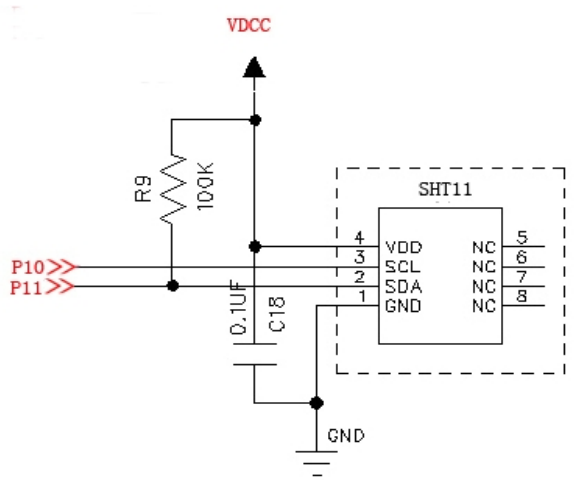

Fig.5 The interface design

This paper selects SHT11 to measure the human temperature and humidity. SHT11 is a digital sensor of Switzerland Scnsirion company, which is capable of simultaneous measurement of temperature and humidity, through the I2C interface for communication between SHT11 and SHCC6601, as shown in Fig. 5. The SCL port and SDA port of SHT11 are respectively connected with P10-P11 interface of SHCC6601. Between DVCC and GND connected to a decoupling capacitor.

\section{System software design}

When the system is powered on, enter the initialization state, set the parameters by pressing a button. The first set of gender, choose the gender of the monitoring object by the switch button ; press the OK button to enter the monitoring object's height setting mode, by changing the buttons to adjust the height. After setting then press the Enter key, the system into the weight setting mode, set the weight of monitored object in the same way, when you press the Enter key to enter the time setting mode, set the current time and again by the key, press the Enter key, system initialization completed.

The system began to run sequentially after initialization, execution repeatedly into the circulation. During system operation needs to accept and implement the relevant button interrupt action, but also need to monitor the real-time status of the motion object in a short period, so the whole system is composed of sequential execution part, interrupted part, short period monitoring part. The interrupted part of the highest priority, priority followed by periodic monitoring instruction.

\section{Interrupt process design}

In order to improve the system performance, which need to minimize the execution time of interrupt program. The software used some flags to control the program execution. Specific control process is as follows: when the system enters break, first read the key value, determine the key species, control flow graph of interrupt program is shown in Fig. 6. First, if the interrupt key value is equal to the mode key value, then the mode flag is set to 1 , otherwise continue to compare values; if the interrupt key value is equal to the start button value, then the start flag is set to 1 , otherwise continue to compare values; if the interrupt key value is equal to the reset button value, then the reset flag is set to 1 , otherwise continue to compare values; if the interrupt key value is equal to the query button value, then the query flag is set to 1 , then end the interrupt program, and back into the main part of the software before the continuation of the original operation. The system to detect the running state in a short period of time, the human body movement monitoring flag is set through the interrupt button, when the flag is set to perform monitoring tasks, thus avoiding useless monitoring, can reduce the power consumption of the system. 


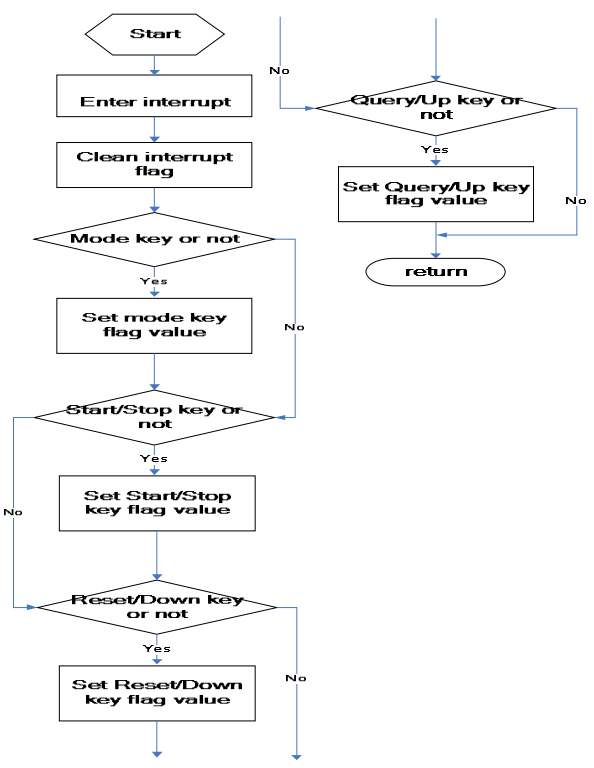

Fig.6 Interrupt process design
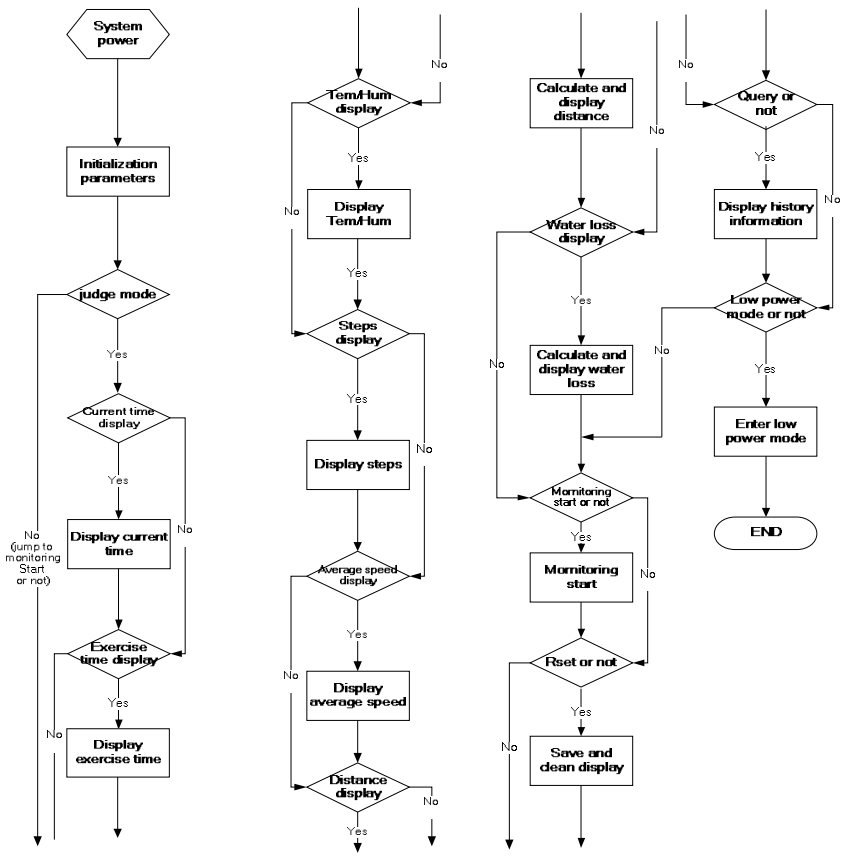

Fig.7 Control flow chart of the

\section{Monitor process design}

Control flow chart of the system is shown in Fig. 7. The control process of the main software is: after the completion of system initialization parameters, the current time printed on a small LCD screen. The main part of the software in order to detect various state.

First judge the mode flag value is zero or not? If the value is not equal to zero, then continue to determine the mode flag bit value, if the value is to show current time mode, then print the current time in the liquid crystal display; if the vale is to show the long time of the motion mode, then print the time in the liquid crystal display; if the vale is to show the current temperature and humidity mode, then drive SHT11 sensor to collect the temperature and humidity and print it in the liquid crystal display; if the vale is to show walking steps, then drive sensor to collect the steps and print it in the liquid crystal display; if the value is to show the average speed of the human, then calculate the speed and print it; if the valve is to show the long of distance, then calculate the distance and print it; if the value is to show the loss of the water, then calculate the loss of the water and print it. Next set the mode flag value to zero. Then continue to determine the monitoring object start flag, if the flag vale is not equal to zero, start to monitor the human motion state. Continue to implement the software, to determine whether the reset flag set? If the reset flag has been set, save the information of last time and remove the information in the liquid display. Next set the reset flag to zero, and read the query flag value, if the value is not equal to zero, then display the history record information.

If all flags value is zero in the course of the processing, the system will enter a low power state.

\section{E Test Result}

Adult males are selected as the monitoring object. After the completion of the initial parameters, the movement monitoring device is fixed at the waist of the monitoring object, the test environment temperature is 25 , the relative humidity is $55.1 \%$, the test time is 35 minutes.Fig. 8 is the number of moving steps, Fig. 9 is the temperature record of the movement process, Fig. 10 is the humidity record of the movement process. Movement after 35 minutes, using third party tools measuring distance is 2436 meters, monitoring device records the number of steps is 3498 and monitoring object stride is $0.7 \mathrm{~m}$, the device calculates the distance: $3498 * 0.7=2448.6$, error is $0.5 \%$. 


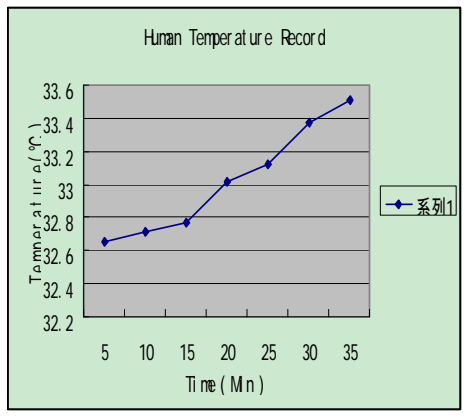

Fig.8 the number of moving steps

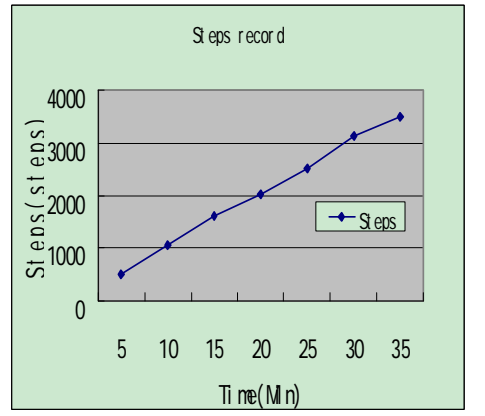

Fig.9 the temperature record

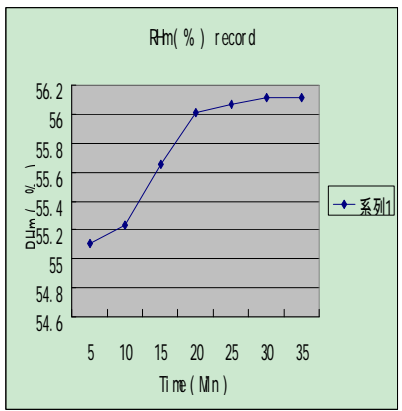

Fig.10 the humidity record

The paper research and design a kind human motion monitoring system. Firstly, completed the design of function modules and the structure of the system, and then describes the hardware design and software design of the core system. Human movement monitoring device has the following characteristics:

a) The system is capable of measuring human gait conditions accurately.

b) The system can measure the body temperature and humidity in the process of movement, which can provide the basis for the calculation object of body water loss.

c) According to the monitoring object parameters input though the keyboard and the human motion time the system can calculate human movement distance and average speed.

d) The system has friendly display interface, and it is very convenience for users to query the state.

e) The system has the characteristics of low power consumption.

The device can realize the real-time monitoring of human movement is good, has a good application value in health care.

\section{Acknowledgment}

The work is supported by the Chongqing college school enterprise cooperation plan ( 143194 ).

\section{References}

[1] Jiang Ke , Ge hong, Zhang Yongbao , Liu Wei , Hu Xiaolin , Lv Xiaodong. Design and implementation of real time multi person movement physiological parameters monitoring system [J] . Medical equipment . 2013(07) : 21-23.

[2] Hong Yan, Yang Min, Chen Yan . Physiological indexes of human body and clothing micro climate monitoring system research and development [J] . Journal of textile.2013 ( 01 ) : 96-100

[3] Qiu Xunchao, Cao Jun. Passive wireless sensor platform in the running application [J]. Computer engineering and design. 2014 ( 12 ) : 17-21.

[4] Guo Hui, Xun Jun, Li Mengjie. The design of the human body parameter acquisition system based on nRF24E1 [J]. Journal of Xi'an Polytechnic University. 2009(08) : 45-49

[5] Cai Yutai, Li Zhefeng, Lin Jiangwei. Bluetooth and smart mobile phone in vivo pulse monitor based on [J]. Medical equipment. 2013(01):13-16

[6] Shanghai Huahong integrated circuit . Datasheet SHC6601[Z] . www.shhic.com/

[7] Variable Timing Injection . Datasheet. CMA3000[Z] . www.21ic.com 
